\title{
Corela
}

Cognition, représentation, langage

HS-2 2005

Le traitement lexicographique des noms propres

\section{Le nom propre en chinois. Essai de morphosyntaxe}

\section{Pierre de La Robertie}

\section{(2) OpenEdition}

\section{Journals}

Édition électronique

URL : http://journals.openedition.org/corela/1187

DOI : $10.4000 /$ corela. 1187

ISSN : 1638-573X

Éditeur

Cercle linguistique du Centre et de l'Ouest - CerLICO

Référence électronique

Pierre de La Robertie, "Le nom propre en chinois. Essai de morphosyntaxe », Corela [En ligne], HS-2 I 2005, mis en ligne le 02 décembre 2005, consulté le 02 avril 2021. URL : http://

journals.openedition.org/corela/1187 ; DOI : https://doi.org/10.4000/corela.1187

Ce document a été généré automatiquement le 2 avril 2021.

\section{(c) (i) (2) (2)}

Corela - cognition, représentation, langage est mis à disposition selon les termes de la licence Creative Commons Attribution - Pas d'Utilisation Commerciale - Partage dans les Mêmes Conditions 4.0 International. 


\title{
Le nom propre en chinois. Essai de morphosyntaxe
}

\author{
Pierre de La Robertie
}

\section{Introduction}

1 Les noms propres forment bien une catégorie de mots reconnus par la linguistique chinoise contemporaine, on les appelle zhuanming 专名. Néanmoins, ils ne font généralement pas l'objet d'un traitement particulier dans les grammaires et autres ouvrages linguistiques. Au mieux, est-il possible de trouver des dictionnaires de noms de personnes, d'œuvres ou de lieux, qui ne sont que des listes ordonnées accompagnées de leur définition... A ma connaissance, seuls les noms de lieux ont fait l'objet d'un traitement particulier, études scientifiques et textes réglementaires. Nous commencerons par présenter quelques caractéristiques de la langue et de l'écriture chinoises, indispensables pour la compréhension de la morphosyntaxe de cette catégorie de mots que sont les noms propres.

\section{La langue et l'écriture chinoises, et la transcription pinyin}

2 Les mots chinois sont invariables. Il n'y a pas, sauf pour les pronoms personnels et quelques noms désignant des personnes, de différenciation de genre et de nombre. Il n'y a pas non plus de désinence verbale permettant de repérer le temps ou le mode, ni le type de sujet du verbe. La langue chinoise ne connaît pas non plus l'article, qu'il soit défini ou indéfini. Ce qui se rapproche le plus de notre article défini, c'est le démonstratif, si le locuteur souhaite l'employer. La grammaire chinoise traditionnelle, avant l'influence des grammaires occidentales avec ses classes de mots, ne connaissait que deux types de mots: les mots pleins ( = chargés de sens: substantifs et verbes d'action et de qualité) et les mots vides (c'est-à-dire tous les mots-outils). 
3 Les mots n'ont pas tous une nature grammaticale déterminée, celle-ci dépend de leur fonction et donc de leur place dans la phrase. L'un des principes fondamentaux dans l'ordre des mots simples et des composants de mots complexes est: le déterminant précède le déterminé. Ainsi, « Pékin est la capitale de la Chine » se dira « Beijing / verbe être / Chine /mot-outil marquant la relation de détermination / capitale » Beijing shi Zhongguo de shoudu ; « le département du Maine-et-Loire » se traduira " Maine-et-Loire / département » Man'en-luaer sheng.

4 En français, les mots - à l'intérieur d'une même phrase - sont séparés les uns des autres par un espace; en chinois, aucun espace ne visualise les différents mots, les sinogrammes se suivent à la queue leu leu, sans que rien ne montre où commence ni où finit le mot. Ce qui signifie que, pour lire un texte, un certain niveau de compréhension et donc de connaissance de la langue, est déjà requis. Prenons un exemple :

鲁迅是中国现代著名的文学家和思想家，绍兴人，生于一八八一年九月二十五

日, 原名周树仁。

5 Le lecteur a remarqué la ponctuation, d'usage assez récent en Chine ; la typographie traditionnelle ne connaissait aucune ponctuation! Rien ne permet de visualiser les différents mots et la nature grammaticale de ceux-ci si l'on ne connaît pas la langue et l'écriture. Reprenons la phrase précédente en insérant des signes séparant les différents mots ou groupes de mots :

鲁迅/是/中国/现代/著名/的/文学家/和/思想家，绍兴/人，生/于/一八八一年/九 月/二十五日, 原/名/周/树仁。

6 D'autre part, il n'y a pas de majuscule pour marquer le début d'un nom propre, comme en français. Le repérage d'un nom propre ne peut se faire que grâce au contexte de la_phrase : il est évident que la position de complément derrière un verbe qui signifie "aller à", "être à", ne peut renvoyer qu'à un toponyme (ou une expression locative).

Exemples :

\begin{tabular}{|l|l|l|}
\hline 我们去重庆 & wŏmen qù Chóngqìng & "nous aller Chongqing" \\
\hline 他不在昆明 & tā bú zài Kūnmíng & "lui pas être-à Kunming" \\
\hline 李洪东在他叔叔那儿 & Li Hóngdōng zài tā shūshu nàr & "li hongdong être-à oncle locatif" \\
\hline
\end{tabular}

7 Devant le mot人, qui signifie " personne, être humain », on peut avoir - en raison du contexte - un toponyme désignant le lieu d'où cette personne est originaire, comme dans cet exemple la ville de Shaoxing 绍兴. De même, le sujet du verbe, souvent placé en position de thème, est fréquemment un mot désignant une personne (nom propre, pronom personnel, appellation...). Dans notre exemple ci-dessus, 鲁迅 est le sujet du verbe 是. Il n'y a pas de différence graphique entre un nom propre et l'adjectif correspondant. Ainsi, 中国 Zhongguo peut se traduire, selon le contexte, par «la Chine» ou "chinois». Dans notre exemple, Zhongguo est déterminant des noms wenxuejia et sixiangjia, qui signifient respectivement "homme de lettres» et " penseur », il est donc à traduire par un adjectif.

8 Une telle écriture, dans laquelle chaque graphème correspond - sur le plan phonologique - à une syllabe, le plus souvent composée d'une consonne initiale suivie d'une voyelle, elle-même pouvant être nasalisée ou suivie de la consonne [n] et à une 
unité sémantique, pose des problèmes de transcription. Pour des raisons évidentes, il ne peut être question de translittération. Les noms propres sont, pour la plupart, constitués de plus de deux ou trois syllabes, donc de deux ou trois sinogrammes. Pendant longtemps, les aventuriers et autres missionnaires se sont débrouillés comme ils pouvaient; puis, des transcriptions quasi-officielles pour les grandes langues (anglais, français, allemand ...) ont vu le jour. A la fin des années 50, le gouvernement de la nouvelle République populaire de Chine a adopté une transcription en lettres latines, tout d'abord à usage interne: alphabétiser les "masses populaires" et les minorités. Cette transcription est couramment appelée "pinyin». Le mot pinyin est l'abréviation de l'expression hànyŭ pinyin zìmŭ, qui signifie "alphabet phonétique du chinois". Il existe des règles d'écriture en pinyin, qui font l'objet de publications destinées au public chinois. Lors de son admission à l'ONU, la Chine a demandé que le pinyin soit reconnu comme système officiel de transcription du chinois dans les organismes internationaux. Très vite, la grande majorité des organes de presse, auteurs et éditeurs l'ont à leur tour employé. C'est donc la transcription que nous employons. Cette transcription est, sur le modèle de l'écriture de nos langues occidentales, morphémique. D'autre part, les linguistes chinois à l'origine du pinyin ont aussi décidé d'employer des majuscules au début des noms propres. C'est ainsi que nous allons écrire notre exemple précédent de la façon suivante: Luxun shi Zhongguo xiandai zhuming de wenxuejia he sixiangjia, Shaoxingren, sheng yu yibabayi nian jiuyue ershiwu ri, yuanming Zhou Shuren et nous traduirons: "Luxun est un homme de lettres et un penseur contemporain chinois, originaire de Shaoxing, il est né le 25 septembre 1881, son nom était Zhou Shuren ${ }^{1} »$.

Un dernier point, important, est l'existence de tons dans la langue chinoise. La langue officielle de la République populaire de Chine, que nous avons pris l'habitude d'appeler "le mandarin», compte quatre tons, représentés en pinyin par quatre signes diacritiques placés au-dessus de la voyelle (ou de la voyelle principale dans le cas des diphtongues). Exemples : ā á ă à. Il faudrait, en raison de nombreuses homophonies, transcrire les mots chinois en tenant compte de ces signes. Néanmoins, pour des raisons pratiques tenant à l'état des logiciels informatiques, ces marques tonales sont souvent absentes des textes, sauf dans ceux à valeur didactique comme les manuels d'apprentissage de la langue.

\section{Les toponymes}

10 Le premier problème est d'ordre méthodologique : comment, dans quelles sources (orales, écrites), appréhender le phénomène toponymique, en particulier la morphosyntaxe des toponymes? La deuxième question est celle de la signification des toponymes, car ils sont formés de mots qui ont une signification. Néanmoins, nous ne traiterons ici que de la morphosyntaxe des toponymes en chinois, laissant de côté cette question -pourtant essentielle- de leur sens.

11 Un rapide survol de sources différentes montre une réelle variété des formes selon le contexte: type de texte, mais aussi dans le même livre - par exemple - selon l'environnement (légende de photo, carte, texte lui-même). La variété formelle réside surtout dans l'emploi ou non de l'appellation générique.

12 Il suffit pour s'en convaincre de regarder la liste des noms de lieux relevés dans le volume Taishun de la collection Xiangtu Zhongguo (que l'on peut traduire par « la Chine 
rurale »), que nous avons choisi d'analyser en détail, car il parle de l'habitat traditionnel dans un district méridional de la province du Zhejiang; nous étions sûr d'y trouver quantités de toponymes. On trouve, par exemple, Baizhang et Baizhang zhen, Daxiyuan et Daxiyuancun, Fujian, Fujian diqu et Fujian sheng, etc., dans lesquelles zhen, cun, diqu et sheng sont des appellations génériques. Zhen est habituellement traduit par le terme de bourg, il désigne une agglomération rurale, la plus petite unité administrative urbaine, souvent également chef-lieu de canton ou de district. Cun désigne un village, plus proche de notre hameau ou du «village naturel» que de notre commune. Diqu correspond à notre région, avec la même imprécision dans sa nature. Sheng est une province, une des unités administratives chinoises de rang 1 , les autres étant la région autonome et la municipalité 2 . Les binômes et trinôme ci-dessus désignent à chaque fois le même objet géographique, à savoir [le bourg de] Baizhang, [le village de] Daxiyuan et [la province du] Fujian. De même, en français, il nous arrive de parler de l'Ille-et-Vilaine ou du département d'Ille-et-Vilaine, de Tours ou de la ville de Tours, etc. en fonction du contexte.

\subsection{Endonymes}

Selon la terminologie des Nations Unies, on appelle endonyme «l'appellation toponymique exprimée dans l'une des langues locales de la région où l'entité dénommée est située $»^{3}$ et exonyme "le nom géographique utilisé dans une langue pour désigner un accident géographique situé en dehors du territoire dont cette langue est la langue officielle, et de forme différente de son nom dans la ou les langues officielles du territoire où il est situé $»^{4}$. Pour prendre un exemple, Paris est l'endonyme de la capitale française, tandis que Parigi est l'exonyme italien correspondant; de même Pékin est l'exonyme français correspondant à l'endonyme Beijing 北京.

En théorie (c'est-à-dire selon les normes réglementaires actuelles édictées par le Bureau de Toponymie, qui dépend du Ministère des Affaires civiles de la République populaire de Chine), les toponymes sont constitués de deux composants :

- l'appellation générique (Ag) tōngmíng 通明

- l'appellation spécifique (As) zhuānmíng 专名.

L'appellation générique, ou élément générique pour reprendre l'expression recommandée par le groupe d'experts sur les noms géographiques des Nations unies, est «la partie d'un toponyme qui désigne de façon générale la nature de l'entité géographique dénommée $»^{5}$. Par exemple, montagne, rivière. L'appellation spécifique, ou élément spécifique selon la terminologie des Nations Unies, est "la partie d'un toponyme qui n'est pas un terme générique, et qui distingue l'entité géographique dénommée des autres appartenant à la même classe $»^{6}$. Exemples: Cap de BonneEspérance, Rio negro.

Exemples :

- Guăngdōng Shěng 广东省

• Luófú Shān 罗浮山 dans lesquels shěng et shān sont des appellations génériques.

L'ordre des composants suit l'ordre général des mots dans les mots composés comme dans les expressions formés d'un nom et d'un ou plusieurs déterminant(s) de nom. Pour les toponymes, l'appellation spécifique précède l'appellation générique. Le nom individuel du lieu (ville, lac, pont, etc.) est considéré comme déterminant le lieu en tant que générique. Il y a autant d'appellations génériques que de types de réalités à 
nommer, et même plus encore, car les façons de nommer les sommets, les cours d'eau, les villages, etc. sont extrêmement variées. il y a quarante-quatre appellations génériques désignant les montagnes, vingt-et-une désignant les cours d'eau, seize désignant les cols et passes, vingt-deux pour les lacs, onze pour les vallées et ravins, seize pour les mers, vingt-deux pour les bancs de sable, vingt-cinq pour les îles et récifs, onze pour les caps, sept pour les "voies d'eau", quatre pour les embouchures, soixantetreize pour les villages!

La quasi totalité des appellations génériques est constitué d'une seule syllabe (i.e. un seul sinogramme).

Exemples :

shĕng 省 "province", xiàn 县 "district", xiāng 乡 "canton", shì 市 "ville", zhēn 镇 "bourg" ; 河 hé "fleuve, rivière", jiāng 江 "fleuve, rivière", $x i$ 溪"rivière"; shān 山 "montagne", fēng 峰 "pic"; qiáo 桥 "pont", lóu 楼 "bâtiment à étages", ling 岭

"chaîne de montagnes", etc.

Mais, on peut rencontrer des disyllabes (dìqū 地区 "région", shānmài 山脉 "chaîne de montagnes", hăixiá 海峡 "détroit", shānkŏu 山口 "col", yùnhé 运河 "canal", ...) et des mots ou expressions de trois syllabes ou plus (zizhìqū 自治区 "région autonome").

Ces appellations génériques composées sont soit constituées d'un seul mot (dì̄ū 地区) soit de deux mots en relation déterminant-déterminé : shān/mài 山脉 "montagne/flux", shān/kŏu 山口 "montagne/ouverture", zizhi/qū 自治区 "autonome/région" ...

De nombreuses appellations spécifiques sont ainsi composées :

• Adjectif + nom. Ex. : Xià/hóng 下洪 "en bas/grande eau" ; Shàng/hóng 上洪 "en haut/grande eau".

•Nom + nom. Ex. : Běi/jīng 北京 "nord/capitale", Quán/zhōu 泉州 "source/territoire", Hú/zhái 胡寨 "famille Hu/demeure", Lóng/tán 龙潭 "dragon/étang"

- Adjectif numéral + nom : Ex. : jiü/jiāng 九江 "neuf/fleuves", Wü/jiā 五家 "cinq/familles". Quant au nombre de syllabes, on peut trouver plusieurs cas (As + Ag) :

• 1+1 : Tài Shān 泰山 Mont Tai

- 2+1 : Dòngtíng Hú 洞庭湖 Lac Dongting

-2+2 : Zhōushān Qúndăo 舟山群岛 Archipel Zhoushan

- Ces trois cas sont les plus fréquents, mais on trouve aussi :

-3+1 : Qīlăotú Shān 七老图山 Mont Qilaotu

-3+2: Bějišshān Lièdăo 北鹿山列岛 Îles Beijishan (dans ce cas, bei «nord» fonctionne comme déterminant de jishan)

-4+1: Zhūmùlángmă Fēng 珠穆朗玛峰 Everest

-4+2 : Hūlúnbèier Căoyuán 呼伦贝尔草原 Steppe de Hulun Boir

• 5+2 : Tăkĕlāmăgān Shāmò 塔克拉玛干沙漠 Désert du Taklamakan.

Certains noms, en particulier de constructions, peuvent être encore plus longs et plus complexes.

Il existe des sortes d'abréviations toponymiques, parfois très différentes du nom propre, pour les noms de province et de grandes villes, en particulier.

Exemples :

\begin{tabular}{|c|c|c|c|}
\hline $\bar{A} n h u \bar{\imath}$ & 安徽 & \begin{tabular}{|l|} 
易 \\
\end{tabular} & Wăn 皖 \\
\hline Bĕijīng & 北京 & 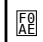 & Jīng 京 \\
\hline
\end{tabular}




\begin{tabular}{|l|l|l|l|}
\hline Húnán & 湖南 & 咂 & Xiāng 湘 \\
\hline Shànghăi & 上海 & 垢 & Hù 沪 \\
\hline
\end{tabular}

Les noms des voies routières et ferroviaires, de régions géographiques ou administratives, emploient fréquemment des abréviations.

Exemples :

\begin{tabular}{|c|c|c|}
\hline Chuān-Qián Xiàn & 川黑线 & $\begin{array}{l}\text { la ligne Sichuan-Guizhou (qian est la dénomination courte de } \\
\text { Guizhou) }\end{array}$ \\
\hline Băo-Chéng Tiélù & 宝成铁路 & la voie ferrée Baoji-Chengdu \\
\hline $\begin{array}{l}\text { Jing-Jìn Gāosù } \\
\text { Gōnglù }\end{array}$ & $\begin{array}{l}\text { 京津高速公 } \\
\text { 路 }\end{array}$ & l'autoroute Beijing-Tianjin \\
\hline Yún-Guì Gāoyuán & 云贵高原 & le plateau du Yunnan-Guizhou \\
\hline
\end{tabular}

Normes d'écriture des toponymes en pinyin:

21 En 1984, est paru un décret sous l'égide de la Commission toponymique chinoise et de la Commission chinoise pour la réforme de l'écriture: "Règles d'écriture en pinyin des toponymes de Chine".

- Lorsque l'on peut identifier appellation spécifique et appellation générique, les deux sont écrites séparées par un espace, avec une majuscule à l'initiale de chacune.

- Les noms de villes et villages ne distinguent pas l'appellation spécifique zhuanming et l'appellation générique tongming dans l'écriture morphémique. De même, les noms des "petits" lieux (xiao diming) sont écrits sans séparer l'appellation spécifique et l'appellation générique, ou qui a perdu sa réalité de générique.

Ainsi, on écrit :

\begin{tabular}{|l|l|}
\hline Hēilóngjiāng Shěng & shĕng signifie "province" \\
\hline $\begin{array}{l}\text { Běijīng Shì } \\
\text { Shíiiāzhuāng Shì }\end{array}$ & shì signifie "ville" (souvent traduit par "municipalité") \\
\hline $\begin{array}{l}\text { Tōng Xiàn } \\
\text { Lánkăo Xiàn }\end{array}$ & xiàn est traduit par "district" (county, en anglais) \\
\hline Hăidiàn Qū & $\begin{array}{l}\text { qū signifie "arrondissement" lorsque c'est l'unité administrative urbaine } \\
\text { dépendant d'une municipalité shi }\end{array}$ \\
\hline Shidu Xiàng & xiàng est traduit par "canton" \\
\hline $\begin{array}{l}\text { Guăngxī Zhuàngzú } \\
\text { Zizhìqū }\end{array}$ & zhuàngzú signifie "ethnie Zhuang", zizhìqū signifie "région autonome" \\
\hline Tàipíng Jiē & jiē signifie "rue" \\
\hline
\end{tabular}




\begin{tabular}{|l|l|}
\hline Pŭtuó Shān & shān signifie "montagne" \\
\hline Táiwān Hăixiá & hăixiá signifie "détroit" \\
\hline Huáng Hé & hé signifie "fleuve" \\
\hline
\end{tabular}

mais :

\begin{tabular}{|l|l|}
\hline Zhōukŏudiàn & diàn signifie "boutique, échoppe" \\
\hline Huŏxiàn & xiàn signifie "district" \\
\hline Wángcūn & cūn signifie "village" \\
\hline Xifengzhēn & zhēn signifie "bourg" \\
\hline Yóufángqiáo & qiáo signifie "pont" \\
\hline
\end{tabular}

- L'initiale d'un toponyme s'écrit en majuscule. Lorsque le toponyme comprend plusieurs éléments, l'initiale de chaque élément prend la majuscule.

- Lorsque les noms de régions sont suivis d'une indication par rapport aux points cardinaux, cette indication n'est pas considérée comme un morphème à part entière, le toponyme est alors écrit sans espace de séparation.

Exemples :

\begin{tabular}{|l|l|}
\hline Huábĕi & $\begin{array}{l}\text { Huá désigne "la Chine" ; běi signifie "nord" Huabei désigne une vaste région septentrionale } \\
\text { de la Chine, qui comprend les provinces du Hebei, du Shanxi, la Région autonome de } \\
\text { Mongolie intérieure et les municipalités de Beijing et Tianjin; }\end{array}$ \\
\hline Huádōng & dōng signifie "est"; \\
\hline Dōngběi & $\begin{array}{l}\text { "Nord-Est" désigne la Mandchourie, constituée de trois provinces, Heilongjiang, Jilin et } \\
\text { Liaoning; }\end{array}$ \\
\hline Jiāngnán & $\begin{array}{l}\text { jiāng signifie "fleuve" ; nán signifie "sud" ; le Jiangnan est une vaste région située au sud } \\
\text { du Changjiang. }\end{array}$ \\
\hline
\end{tabular}

- Lorsqu'un nom de personne fait partie d'un toponyme en tant que appellation spécifique, il est généralement écrit comme un seul morphème; mais, il arrive qu'il continue de faire référence à l'individu et donc s'écrit alors en deux mots. Ex.: Zhangzhidong $L u$ "Rue Zhangzhidong" (Zhang Zhidong est un personnage historique), Sun Zhongshan Jiniantang "Mémorial Sun Yat-sen" (Yat-sen est la transcription de la prononciation cantonaise d'un prénom de l'homme politique dont le nom en mandarin s'écrit Sun Zhongshan).

- Lorsqu'une appellation générique est employée dans un nom de lieu comme élément de l'appellation spécifique, elle n'est pas graphiquement séparée de cette dernière, p.ex.: Heilongjiang Sheng "Province du Heilongjiang", dans laquelle jiang "fleuve" est appellation générique du fleuve nommé Heilong (Jiang). 
- Les noms de villages ou autres "petits" lieux dont le nom se termine par un mot qui fut autrefois générique, mais dont la référence à la réalité a disparu, s'écrivent en un seul mot, sans séparation. On trouve souvent comme syllabe finale : cūn "village", zhēn "bourg", diàn "auberge", pŭ "boutique", băo "fort", qiáo "pont", miāo "temple", jí "marché, jĭng "puits", lóu "bâtiment à étages", kŏu "bouche, ouverture, embouchure", yíng "garnison", chăng "marché de plein air" et quelques autres.

22 Attention : des mots différents peuvent s'écrire avec la même transcription pinyin, surtout lorsque les marques tonales sont absentes, ce qui est souvent le cas, surtout sur les cartes; on ne peut donc pas déduire de la transcription une signification certaine du mot, hors contexte. Par exemple, Changning Xian peut désigner un district dans la province du Yunnan, dans celle du Hunan ou dans celle du Sichuan, mais les trois noms s'écrivent différemment en sinogrammes: 昌宁县 dans le Yunnan, 常宁县 dans le Hunan, 长宁县 dans le Sichuan. Un même caractère (ou un mot écrit en sinogrammes) a un sens, même si Chinois de Cantonais, de Shanghai, ou d'ailleurs prononcent différemment selon leur dialecte (ou langue) materne(le), comme d'ailleurs un Japonais ou un Coréen. Par exemple, 山se prononce shān en mandarin, san en cantonais, en sinocoréen et en sino-japonais, yama en japonais (lecture "yamato"), mais signifie toujours "montagne".

\subsection{La réalité}

La réalité toponymique, telle que nous l'avons rencontrée dans des atlas, des livres et des revues, est quelque peu différente des normes décrites précédemment. Le principal écart d'avec les normes concerne l'emploi des appellations génériques.

- Les appellations génériques qui sont traduites en français par les termes "province", "ville" (ou "municipalité") sont souvent élidées dans les textes, mais figurent généralement sur les cartes et atlas.

- Les appellations génériques traduites par les termes "bourg", "village", sont souvent élidées dans les atlas et sur les cartes, mais elle figurent dans les textes.

- Les appellations génériques traduites par les termes "fleuve", "rivière", "mont" ou "montagne", "chaîne de montagne", "désert", et autres termes géographiques, sont généralement présentes.

- Les appellations génériques dans les noms de constructions, sites, usines et autres réalités humaines, sont généralement présentes.

Pour les réalités liées à l'habitat, le contexte semble jouer un rôle important voire essentiel : lorsque l'on est dans un contexte administratif, l'appellation générique est présente.

A la lecture du livre Táishùn 泰顺, de la collection Xiangtu Zhongguo, nous avons remarqué que :

1. Sur les cartes,

- les noms des provinces sont employés sans appellation générique, tandis que les noms des municipalités de rang provincial et de rang préfectoral se terminent par l'appellation générique shì 市 qui signifie « municipalité, ville »,

- les noms de toutes les villes comportent l'appellation générique shì 市,

- les noms de tous les cours d'eau se terminent par les appellations génériques hé 河, jiāng 江 ou $x \bar{i}$ 溪, qui toutes signifient «fleuve ou rivière », 
- les noms de tous les reliefs se terminent par l'appellation générique shān 山 «mont, montagne",

- les noms des "bourgs" ou petites villes rurales, souvent chefs-lieux de canton ou de district, se terminent par l'appellation générique zhēn 镇,

- mais, les noms des chefs-lieux de district sur la carte provinciale ne sont pas suivis d'une appellation générique,

- les noms des villages et petites agglomérations ne sont pas suivis d'une appellation générique sur la carte du district.

2. Sur les photos, les légendes comportent toujours des appellations génériques : xiāng 乡 « canton », zhēn 镇 « bourg ", le nom de la construction (pont, etc.).

3. L'appellation générique xiàn 县 «district» est mentionnée lorsqu'il s'agit de la création de l'unité administrative.

Quelques types d'énoncé (les appellations génériques sont soulignées et nous n'avons pas employé les marques tonales dans ces exemples):

-Yongjia, Pingri, Taishun he Ruian si xian shi 永嘉, 平日, 泰顺和瑞安四县市

- « Les quatre districts et municipalités de Yongjia, Pingri, Taishun et Ruian »

- Shanghai Tongji Daxue he Jiaotong Daxue 上海同济大学和交通大学

- «L'Université Tongji et l'Université Jiaotong de Shanghai »

- Zhejiang de Jingning, Taishun er xian 浙江的景宁, 泰顺二县

- « Les deux districts de Jingning et de Taishun au Zhejiang »

- Fujian Sheng de Shouning Xian 福建省的寿宁县

- «Le district de Shouning dans la province du Fujian »

- Feiyun iang, Jiao Xi， Shaocheng Gang, Ao \iang si da shuixi 飞云江，交溪，少坮港，鳌江四 大水系

- « Les quatre cours d'eau Feiyun Jiang, Jiao Xi , Shaocheng Gang et Ao Jiang »

- Luoyang, Sankui, Sixi deng pendi 洛阴, 三鬼, 泗溪等盆地

- «Les plaines de Luoyang, Sankui et Sixi »

- Fujian Shouning, Ningde,Putian,Changxi he Fuding deng di 福建寿宁, 宁德, 普天, 长溪和 福鼎等地

- «Shouning, Ningde, Putian, Changxi et Fuding au Fujian »

- Zhe Min 浙闽 «Zhejiang et Fujian»

-Jingning, Shouning, Zherong,Qingyuan deng qi ge xian 景宁，寿宁，柘荣，庆元等七个县

- « Les sept districts Jingning, Shouning, Zherong, Qingyuan etc. »

- Jiangxi Jiujiang 江西九江

• « Jiujiang au Jiangxi »

- Siqian yu Hongkou liang $\underline{x i}$ 司前与洪口两溪

- «Les deux rivières Siqian et Hongkou »

Certains villages n'ont pas d'appellation générique :

- soit parce qu'il n'y en a pas : 王福 Wangfu, 景中 Jingzhong, 解放 Jiefang,

- soit parce qu'elle fait partie intégrante du nom propre : 文海 Wenhai, 沙河 Shahe, 青虚山 Qingxushan, 郭家街 Guojiajie.

On peut alors ajouter le mot 村cún "village" à ces toponymes, comme cela a été fait dans la base de données de la carte nationale du relief. 


\subsection{Exonymes}

Comment sont traduits/transcrits en chinois les noms propres originellement en langues non-chinoises? La question est la même, bien qu'en des termes quelque peu différents, pour les langues parlées hors de Chine (français, anglais, etc.) et celles parlées en Chine par les ethnies minoritaires (tibétain, mongol, ouïgour, etc...).

Une norme a été publiée en juin 1976, par le Bureau national topographique de la République populaire de Chine et la Commission nationale de réforme de l'écriture, pour la transcription en pinyin des toponymes en langues minoritaires. Les langues parlées par les minorités qui vivent en Chine posent plusieurs problèmes: premièrement, lorsqu'elles ne sont pas écrites, il convient de créer une écriture, généralement en caractères latins; deuxièmement, lorsqu'elles sont écrites, il faut adopter une transcription ou une translittération en lettres latines, comme le pinyin.

Dans les deux cas, une question se pose : comment rendre en sinogrammes les noms propres, parmi lesquels les toponymes? Faut-il les transcrire phonétiquement et donc trouver des sinogrammes qui, sans doute imparfaitement, permettraient de rendre peu ou prou la prononciation locale? Ou bien traduire, quand c'est possible? La transcription en lettres latines doit-elle suivre la transcription chinoise pinyin ou bien doit-elle procéder directement à partir de la langue source ? La réponse à ces questions est certes d'ordre linguistique, mais aussi politique, comme le montre l'examen des cartes et atlas publiés en République populaire de Chine depuis 1960 environ.

- Les toponymes en langues "minoritaires" sont écrits en séparant l'appellation spécifique et l'appellation générique.

- Les toponymes en langues "minoritaires" peuvent être soit transcrits soit traduits, ou encore en partie transcrits en partie traduits, mais ils suivent toujours les habitudes du pinyin en chinois. Les parties traduites s'écrivent en suivant la notation phonétique des sinogrammes ( = en pinyin), les parties transcrites s'écrivent selon la langue "minoritaire".

Le nom de la capitale de la Région autonome du Xinjiang peut s'écrire Wulumuqi, qui est la transcription pinyin correspondant aux sinogrammes (乌鲁木齐) utilisés pour écrire en chinois le nom ouigour (une langue turque-orientale) de cette ville, que l'on peut aussi écrire Ürümqi, en respectant la translittération du ouïgour.

Les exemples ci-après sont écrits selon l'ordre suivant de gauche à droite ; le toponyme en langue minoritaire en écriture latine, la transcription en sinogrammes et la transcription pinyin des sinogrammes. En outre, nous avons laissé de côté l'appellation générique.

Exemples de toponymes ouïgours :

\begin{tabular}{|l|l|l|}
\hline Karamay & 克拉玛依 & Kelamayi \\
\hline Hotan & 和田 & Hetian \\
\hline Kumul & 哈密 & Hami \\
\hline Kaxgar & 喀什 & Kashi ( = Kashgar) \\
\hline
\end{tabular}

Exemples de toponymes mongols : 


\begin{tabular}{|l|l|l|}
\hline Hohhot & 呼和浩特 & Huhehaote \\
\hline Hailar & 海拉尔 & Hailaer \\
\hline Xilin Gol & 锡林郭勒 & Xilin'guolei \\
\hline Ulanqab & 乌兰察布 & Wulanchabu \\
\hline
\end{tabular}

Exemples de toponymes tibétains :

\begin{tabular}{|l|l|l|}
\hline Lhasa & 拉萨 & Lasa \\
\hline Qamdo & 昌都 & Changdu \\
\hline Xigazê & 日喀则 & Rikaze \\
\hline Nyingchi & 林芝 & Linzhi \\
\hline $\begin{array}{l}\text { Yarlung } \\
\text { Zangbo }\end{array}$ & $\begin{array}{l}\text { 雅鲁藏 } \\
\text { 布 }\end{array}$ & $\begin{array}{l}\text { Yaluzangbu (Jiang) Jiang est une appellation générique, qui signifie } \\
\text { «fleuve ». }\end{array}$ \\
\hline
\end{tabular}

Les toponymes étrangers sont de deux types selon l'écriture utilisée : au Japon, mais aussi en Corée et -anciennement- au Vietnam, ils sont écrits en sinogrammes; ailleurs, ils sont écrits dans le système d'écriture en usage dans le pays (alphabet latin, alphabet cyrillique, alphabet arabe, etc.). Pour la première catégorie d'exonymes, il n'y a aucune modification d'écriture entre un texte japonais et un texte chinois, seule la prononciation change, les locuteurs les prononçant chacun dans leur langue (le principe est le même pour les noms de personnes).

Les exemples ci-après sont ainsi écrits, de gauche à droite: le toponyme en sinogrammes tels qu'au Japon ou en Corée, la transcription des sinogrammes en pinyin, la transcription française courante selon l'alphabet latin, la traduction du nom " chinois ». Nous avons délaissé les appellations génériques. Les sinogrammes employés au Japon et en Corée peuvent différer de ceux utilisés en Chine populaire, car les autorités chinoises ont procédé à une simplification de l'écriture au cours des années 60 et 70, qui ont modifié la graphie d'environ deux mille caractères.

Exemples :

\begin{tabular}{|l|l|l|l|}
\hline 東京 & Dongjing & Tokyo & "capitale orientale" \\
\hline 北海道 & Beihaidao & Hokkaido & "province maritime septentrionale" \\
\hline 仙臺 & Xiantai & Sendai & "terrasse aux immortels" \\
\hline 京都 & Jingdu & Kyoto & "capitale" \\
\hline 富士山 & Fushishan & Fujiyama & "mont Fuji" \\
\hline 宫城 & Gongcheng & Miyagi & "ville-palais" \\
\hline
\end{tabular}




\begin{tabular}{|l|l|l|l|}
\hline 漢城 & Hancheng & Séoul & "ville (sur le fleuve) Han" \\
\hline
\end{tabular}

En ce qui concerne les exonymes "vrais", l'habitude est de les transcrire phonétiquement et de les écrire en sinogrammes sans tenir compte du sens de ceux-ci, surtout les appellations spécifiques. Il n'est pas rare que le toponyme étranger passe par le truchement de la langue anglaise avant d'être transcrit en chinois, ce qui ajoute à la déformation due à la transcription. Dans les exemples ci-dessous, les trois colonnes sont dans l'ordre suivant : la transcription en sinogrammes, la transcription pinyin des sinogrammes et le nom dans la langue d'origine.

Exemples :

\begin{tabular}{|l|l|l|}
\hline 柏林 & Bolin & Berlin \\
\hline 纽约 & Niuyue & New-York \\
\hline 诺曼底 & Nuomandi & Normandie \\
\hline 斯德哥尔摩 & Sidegeermo & Stockholm \\
\hline 米兰 & Milan & Milano \\
\hline 佛洛伦萨 & Foluolunsa & Firenze \\
\hline
\end{tabular}

Les appellations génériques ayant un sens et celui-ci étant nécessaire à la compréhension $\mathrm{du}$ toponyme, elles sont traduites en chinois par l'appellation générique correspondante, et parfois même ajoutées au toponyme originel. Dans les exemples, les appellations génériques sont soulignées.

Exemples :

\begin{tabular}{|l|l|l|}
\hline 侏罗山 & Zhuluo $\underline{\text { Shan }}$ & Jura \\
\hline 勃朗山 & Bolang Shan & Mont Blanc \\
\hline 幼发拉底河 & Youfaladi $\underline{\mathrm{He}}$ & Euphrate \\
\hline 的的喀喀湖 & Didikaka $\underline{\mathrm{Hu}}$ & lac Titicaca \\
\hline 百慕大群岛 & Baimuda Qundao & archipel des Bermudes \\
\hline
\end{tabular}

Shan signifie "montagne", he signifie "fleuve, rivière ", hu signifie «lac » et qundao signifie « archipel» (dao a le sen d' « île » et qun est une sorte de préfixe marquant la multiplicité).

Néanmoins, il existe également des parties d'appellations spécifiques (lorsque celles-ci sont composées) qui ont un sens et qui se prêtent à traduction; il arrive alors, mais ce n'est pas systématique, que ces mots soient traduits en chinois, on obtient alors un toponyme mixte traduit-transcrit. Nous avons souligné, dans les exemples, les parties 
traduites des toponymes, et nous avons traduit deux noms de villes universitaires britanniques. Remarquons que le mot chinois bao traduit les mots français bourg, allemand burg ou espagnol alcalá, qui désignent les mêmes réalités.

Exemples :

\begin{tabular}{|l|l|l|}
\hline 汉堡 & Hanbao & Hamburg \\
\hline 约翰内斯堡 & Yuehanneisibao & Johannesburg \\
\hline 雷亚尔堡 & Leiyaerbao & Alcalá la Real (Espagne) \\
\hline 孔特堡 & Kongtebao & Bourg-des-Comptes (Ile-et-Vilaine, France) \\
\hline 黑海 & $\underline{\text { Hei Hai }}$ & mer Noire \\
\hline 死海 & $\underline{\text { Si Hai }}$ & mer Morte \\
\hline 威尔士亲王岛 & Weiershi Qinwang Dao & $\underline{\text { Prince of Wales Island }}$ \\
\hline 新南威尔士州 & $\underline{\text { Xinnan Weiershi Zhou }}$ & State of New $\underline{\text { South Wales }}$ \\
\hline 剑桥 & Jianqiao & "épée-pont" Cambridge \\
\hline 牛津 & Niujin & "bœuf-gué" Oxford \\
\hline
\end{tabular}

41 Hai veut dire " mer ", bao désigne une ville fortifiée, dao est une " île "; zhou désigne un territoire, une unité administrative, ce terme est employé pour traduire le terme étranger d'état, en Australie, aux Etats-Unis, etc.

Certains lieux étrangers ont un nom chinois, différent du nom étranger.

Exemples :

\begin{tabular}{|l|l|l|l|}
\hline San Francisco & 旧金山 & Jiujinshan & “vieille/or/montagne” \\
\hline Honolulu & 檀香山 & Tanxiangshan & “santal/montagne” \\
\hline
\end{tabular}

\section{Les noms de personne}

\subsection{Les noms chinois}

Conformément à notre présentation de la langue et de l'écriture chinoises en début de cet article, rien ne permet de distinguer formellement que tel ou tel ensemble de sinogrammes désigne une personne et qu'il s'agit d'un nom propre. Comme pour les noms de lieux, seul le contexte et la connaissance que le lecteur a de la langue lui permet de repérer un nom de personne. Il arrive fréquemment que ce nom soit précédé ou suivi d'une expression qui donne sa fonction, son titre, sa relation à une autre personne précédemment citée. L'emploi d'un mot de deux ou trois caractères comme 
sujet d'un verbe d'action est aussi un indice que ce mot est sans doute un nom de personne, ou encore sa fonction comme complément de tel ou tel verbe ...

Donnons quelques exemples tirés de la presse et d'un recueil d'enquêtes sur le monde rural.

1. 省农经委副主任的吴昭仁, 接受了。。 sheng nong jing wei fuzhuren de Wu Zhaoren

2. 管理处处长李显刚和副处长黄炜 guanlichu chuzhang Li Xian'gang he fuchuzhang Huang Wei

3. 黄晓东主任 Huang Xiaodong zhuren

4. 他告诉吴昭仁 ta gaosu Wu Zhaoren

5. 丁作明的死 Ding Zuoming de si

6. 副县长徐怀棠 fuxianzhang Xu Huaitang

7. 丁继营老人 Ding Jiying laoren

8. 丁作明爱人祝多芳 Ding Zuoming airen Wu Duofang

9. 十二岁的丁卫 shiersui de Ding Wei

Traduction de ces exemples:

1. Wu Zuoren, directeur-adjoint de la commission économique agricole provinciale

2. Li Xian'gang chef de bureau au Bureau de gestion et Huang Wei chef-adjoint

3. Le directeur Huang Xiaodong

4. Il dit à Wu Zhaoren

5. La mort de Ding Zuoming

6. Le chef-adjoint de district Xu Huaitang

7. Le vieux Ding Jiying

8. L'épouse de Ding Zuoming, Wu Duofang,

9. Ding Wei, âgé de 12 ans

43 Il est clair, dans ces exemples, que l'entourage lexical, qu'il s'agisse d'appositions (exemples $2,3,6,7,8$; nous entendons pas là qu'il n'y a aucun mot-outil reliant le nom propre et le mot ou l'expression qui le qualifie), de déterminations (exemples 1, 5, 9), ou encore de verbe dont le complément d'objet est nécessairement un nom désignant une personne (nom commun ou nom propre) ou un pronom personnel (exemple 5 : le verbe gàosu) joue un rôle déterminant dans la reconnaissance d'un mot comme nom propre, en particulier comme nom de personne. Malheureusement pour ceux qui voudraient créer un programme automatique de reconnaissance de noms propres de personne, les exemples dans lesquels rien ne permet formellement de distinguer un nom de personne d'autres types de mots sont extrêmement fréquents.

Le lecteur qui regardera attentivement les exemples ci-dessus en sinogrammes pourra remarquer que rien ne permet, visuellement, de distinguer les mots entre eux ni ceux qui forment des noms propres. La transcription pinyin, que nous avons présentée auparavant, distingue les mots en les séparant par un espace blanc, d'autre part les noms propres commencent tous par une majuscule, ce qui permet un repérage immédiat.

Mais, il est un point important à connaître : la place respective du nom de famille et du nom personnel (ou prénom). En Occident, le prénom précède normalement le patronyme, et ceci est marqué dans le mot "prénom» lui-même. En Chine, comme dans d'autres cultures voisines (Vietnam, Japon, Corée, par exemple), le nom de famille précède toujours l'idionyme. Ainsi, dans les exemples précédents, Wu, Li, Huang, Ding 
et $\mathrm{Xu}$ sont des noms de famille, tandis que Zhaoren, Xian'gang, Wei, Xiaodong, Zuoming, Huaitang, Jiying, Duofang et Wei sont les noms personnels des personnes citées. Il n'existe d'ailleurs qu'un nombre relativement restreint de patronymes en Chine, malgré l'important effectif de la population. L' "Encyclopédie des noms de famille de Chine " ne donne qu'environ trois mille patronymes, et des enquêtes liées aux recensements de ces vingt dernières années montrent que les cent patronymes les plus fréquents concernent $87 \%$ de la population chinoise $! 7,9 \%$ de la population chinoise se nomment Li 李, c'est-à-dire que 102700000 personnes portent ce nom de famille! Le prénom est donc d'une extrême importance pour distinguer les différents individus.

Les exemples précédents, comme une recherche rapide sur Internet (en français et en anglais), permettent de voir que les noms de famille sont pour la plupart monosyllabiques, seuls quelques-uns sont dissyllabiques. L'ensemble nom-prénom est le plus souvent composé de trois syllabes, ce qui veut dire que le prénom est souvent dissyllabique. Prenons quelques exemples dans le monde politique actuel de la Chine: $\mathrm{Hu}$ Jintao, Jiang Zemin, Wu Bangguo, Zeng Qinghong, Jiang Chunyun, etc. Bien sûr, il existe aussi des cas, peu fréquents, où cette règle $1+2$ est délaissée, au profit de structures différentes : 1+1 comme Chen Yun, Wu Yi ou Gong Li, 2+1 comme Sima Yi ou Ouyang Xiu, 2+2 comme Sima Xiangru. Cette dernière est, de loin, la plus rare.

SI l'on hérite de son patronyme, en Chine comme en Europe, le prénom, est, en Chine une création ex nihilo pour tel ou tel enfant. Ce sont les parents, ou une personne compétente et habilitée par eux, qui choisissent le prénom de l'enfant. Il n'y a ni liste des saints, ni grand homme à honorer... Dans le choix des mots entrent en jeu de nombreux éléments, en fait ce sont tous les éléments composant les sinogrammes qui doivent être pris en compte: l'aspect phonique, l'aspect sémantique et l'aspect graphique, dans leurs relations avec l'autre mot qui forme le prénom, mais aussi avec le nom de famille et d'autres critères sociaux, historiques, ou culturels.

Il n'est pas souhaitable que les trois syllabes en jeu commencent par la même consonne, ni par des consonnes de même catégorie. Les consonnes peuvent être classées ainsi en cinq types : $\mathrm{b} \mathrm{p} \mathrm{m} \mathrm{/} \mathrm{d} \mathrm{t} \mathrm{n} \mathrm{l} \mathrm{/} \mathrm{g} \mathrm{k} \mathrm{h} \mathrm{/} \mathrm{z} \mathrm{c} \mathrm{s} \mathrm{/} \mathrm{zh} \mathrm{ch} \mathrm{sh.} \mathrm{Les} \mathrm{allitérations} \mathrm{sont} \mathrm{à} \mathrm{proscrire} \mathrm{:}$ Feng Fufen, Tian Tingtang, Feng Mofen, Tian Dengtang sont ainsi considérés comme inadéquats. Il convient de faire attention aux différences phoniques dues aux accents locaux ou aux dialectes régionaux, qui peuvent provoquer - à l'oral- des confusions de consonnes et donc des méprises. Lorsque la finale du nom de famille est une voyelle (i u ï), il vaut mieux éviter un prénom qui commencerait par une syllabe à consonne initiale vide ; c'est ainsi que Li Ende 李恩德ou Du Anfu 杜安福peuvent être confondus sur le plan sonore avec Lin De 林德et Duan Fu 段福. Lorsque l'on choisit un prénom monosyllabique, il est fréquent de répéter cette syllabe unique, de façon à créer une chaîne sonore agréable, et créer ainsi un prénom dissyllabique. Il convient d'éviter que le ton des trois syllabes soit le même.

La relation sémantique peut être souhaitée : par exemple, le patronyme Tian $⿴ 囗 十$ est aussi un nom commun qui désigne «le champ cultivé », le prénom Jinqiu peut former un ensemble particulièrement faste, s'il est composé des caractères 金 et 秋, qui signifient respectivement "or" et "automne", ce qui donne un ensemble nomprénom que l'on pourrait traduire comme "automne doré dans les champs", à comprendre à la fois comme une expression de la belle couleur jaune des champs de céréales mûres, mais aussi comme une allusion à la richesse qu'apporte une bonne 
moisson! le choix de prénom doit tenir compte du fait que la plupart des noms de famille sont aussi des noms communs ou des adjectifs, brefs qu'ils appartiennent au vocabulaire courant et ont une signification ordinaire, cachée, mais présente. La nature grammaticale (nom, adjectif, verbe...) des mots qui composent le prénom doit aussi être prise en compte.

Les sinogrammes peuvent être répartis en plusieurs catégories selon leur graphisme :

- Simples, indécomposables : 丁 Ding, Z Yi...

- Décomposables en partie supérieure et partie inférieure : 贾 Jia, 裏 Xiang ...

- Décomposables en partie gauche et partie droite : 杨 Yang, 张 Zhang ...

- Décomposables selon une diagonale : 廖 Liao, 右 You ...

- Décomposables en partie externe et partie interne : 周 Zhou, 阎 Yan ...

Il vaut mieux éviter que le patronyme et le prénom soient de même type graphique. Si le patronyme est constitué d'un sinogramme simple, il est préférable que le premier sinogramme du prénom soit complexe et le deuxième de type simple, ce qui crée une harmonie visuelle: par exemple, Liu Hua 刘华 est préférable à Liu Qi 刘祁, Ding Shuntian 丁顺天et Wan Guoquan 万国全procèdent d'une telle harmonie. Par contre, si l'on choisit un prénom monosyllabique, il convient qu'il soit aussi simple que le patronyme, comme Ding Yi 丁一 ou Wan Bao 万宝. Si le patronyme est un sinogramme complexe, alors il vaut mieux que le premier sinogramme d'un prénom dissyllabique soit simple et le deuxième complexe : 翟九德Di Jiude, 霍云雁Huo Yunyan. Par contre, si le prénom est monosyllabique, il est préférable qu'il soit de type graphique complexe, comme le nom de famille : 翟德Di De, 霍薇Huo Wei.

On peut aussi jouer sur l'ensemble nom-prénom, de sorte que juxtaposés les deux sinogrammes en forment un autre (Tian $\mathrm{Yu}$ 田雨qui donnent le caractère 雷 lei 《orage », Wang Baishi 王白石qui donnent le caractère 碧bi « jade bleuté »); on peut aussi procéder par diminution ou augmentation du nombre de traits (Yuan Yuan, Nie Er 聶耳); il est également possible de procéder par multiplication du sinogramme qui constitue le patronyme pour former le prénom (Jin Xin 金金金); on peut encore jouer d'une identité graphique partielle entre nom et prénom (Wang Yang 汪洋, Sha Ting 沙 汀).

52 Les sinogrammes qui composent le prénom peuvent faire allusion à des expressions classiques devenues quasiment proverbiales. Le prénom peut être choisi de sorte qu'il forme avec le nom de famille une référence culturelle, historique ou littéraire : Lu Song 鲁颂 : le patronyme Lu est aussi le nom de la principauté antique, d'où était originaire Confucius, tandis que le prénom Song « hymne religieuse » désigne aussi l'un groupe de textes poétiques dans la plus ancienne anthologie poétique chinoise, dont la légende attribue précisément à Confucius la compilation; l'ensemble nom-prénom peut se comprendre comme signifiant « hymnes [du pays]de Lu». Le prénom peut être choisi pour que l'ensemble qu'il forme avec le patronyme ait un sens: Jiang Wanli 江万里 "Fleuve $10000 \mathrm{li}$ » (le li est une mesure traditionnelle de distance, qui équivaut à environ un demi-kilomètre).

\subsection{Les noms étrangers}

53 Les noms de personne étrangers posent, comme pour les toponymes, un premier problème : la transcription (traduction?) d'un type d'écriture (latine, cyrillique, arabe, 
grecque, etc.) en un autre, logographique. La deuxième question, corollaire de la précédente, est : quels sinogrammes choisir pour cette transcription?

Même si l'on peut imaginer que certains noms ou prénoms étrangers ont une signification aisée à reconnaître (Pierre, Philippe ; Dubois, Dupont ...), il est difficile de demander à tous les Chinois ni même aux intellectuels de connaître la signification étymologique des noms et prénoms de la Terre entière. Ainsi, seule la transcription phonétique apparaît comme une solution. D'autre part, il existe des difficultés qui proviennent de différences phologiques entre le chinois mandarin et les langues à transcrire. Donnons quelques exemples : le prénom Marc est écrit avec deux consonnes finales, la seule possibilité en chinois est de prononcer trois syllabes ma-er-ke, donc d'écrire avec trois sinogrammes. Le patronyme de l'actuel président français, Chirac, se termine par une consonne, chose impossible en chinois, son nom devient xi-la-ke. Se pose alors la redoutable question technique du choix des sinogrammes: on peut imaginer que chaque locuteur chinois, qui rencontre un nom étranger, l'écrit comme il pense devoir le faire, cela donne autant de résultats que d'écrivains. En effet, le nombre de syllabes est assez peu nombreux en chinois : 410, cela signifie que de nombreux sinogrammes sont homophones (à plus forte raison si l'on ne tient pas compte des tons, ce qui est le cas dans les transcriptions de mots étrangers). Il existe ainsi, nous dit Madame Alleton, "vingt-huit transcriptions différentes " pour le nom du chimiste russe Mendeleïev'! C'est la situation habituelle jusqu'à l'époque tout à fait contemporaine. Il convient alors de prononcer à voix audible les sinogrammes reconnus comme constituant un nom propre de personne (comme pour les noms propres de lieu) et de deviner, à l'aide du contexte, de qui il s'agit. Il reste une part importante de « hasard » dans ce jeu, et sans doute les déchets (non-reconnaissance) ne sont-ils pas rares, puisque la reconnaissance dépend beaucoup du niveau culturel et éducatif du lecteur. "Les transcriptions ne sont pas toujours facilement identifiables ${ }^{8}$.

C'est pourquoi, ces dernières années, sont parus des dictionnaires de transcription pour les noms et prénoms français, anglais, etc. , qui ont pour tâche de régulariser la situation. Le but est d'arriver à ce qu'à telle syllabe dans telle langue étrangère corresponde tel caractère chinois. Mais, la normalisation ne va jamais aussi vite que la presse ou les besoins de telle ou telle entreprise.

Comment le lecteur peut-il identifier cet ensemble de sinogrammes comme étant un nom de personne étrangère ? Comment peut-il ensuite en connaître l'identité ?

Quelques exemples tirés de la presse de ces derniers mois :

- 德国总理施罗德 deguozonglishiluode que l'on peut rectifier en Deguo zongli Shiluode

- 法国总统希拉克 faguozongtongxilake que l'on peut rectifier en Faguo zongtong Xilake

- 委会主席巴罗嗦 weihuizhuxibaluosuo que l'on peut rectifier en Weihui zhuxi Baluosuo

-敏科专家帕特立克.奥尔德〔P.HORDE〕 minkezhuanjiapatelike.aoerde que l'on peut rectifier en minke zhuanjia Patelike,Aoerde

-敏科医生奥尔德解释 minkeyishengaoerdejieshi que l'on peut rectifier en minke yisheng Aoerde jieshi

-二十九岁的卡特琳娜 ershijiusuidekatelinna que l'on peut rectifier en 29 sui de Katelinna

- 莱斯施风式访问亚洲 laisishifengshifangwenyazhou que l'on peut rectifier en Laisi shifengshi fangwen Yazhou

- 天主教皇保罗二世 tianzhujiaohuangbaoluoershi que l'on peut rectifier en tianzhu jiaohuang Baoluo Ershi 
•天主教教皇约翰保罗二世在梵蒂冈去世

tianzhujiaojiaohuangyuehan.baoluoershizaifantigangqushi que l'on peut rectifier en tianzhujiao jiaohuang Yuehan.Baoluo Ershi zai Fantigang qu shi

- 约旦外交部长莫其说 yuedanwaijiaobuzhangmoqishuo que l'on peut rectifier en Yuedan waijiao buzhang Moqi shuo

•约旦国王阿布都拉二世、沙乌地阿拉伯领袖阿济兹亲王

yuedanguowangabudulaershi,shawudialabolingxiuajiziqinwang que l'on peut rectifier en Yuedan guowang Abudula Ershi, Shawudi Alabo lingxiu Ajizi qinwang

- 麦卡特尼姐妹。。。罗伯特·麦卡特尼 maikatenijiemei... luobote.maikateni que l'on peut rectifier en Maikateni jiemei... Luobote.Maikateni

Traduction de ces exemples et commentaire :

- Le chancelier allemand Shroeder

- Le président français Chirac

- Le président de la Commission [européenne] Barroso

- L'expert allergologue Patrick Horde

- Le docteur allergologue Horde explique ...

- Catherine de 29 ans

- Rice en visite officielle en Asie

- Le pape catholique Paul II

- Le pape de l'Eglise catholique Jean-Paul II décède au Vatican

- Le ministre des Affaires étrangères de Jordanie Mulki dit

- Le roi de Jordanie Abdullah II et le prince Aziz dirigeant de l'Arabie saoudite

- Les sœurs MacCartney... Robert MacCartney

Comme pour les noms de personnes chinoises, la présence dans un texte du titre, de la fonction, du pays d'origine permet de repérer et de comprendre immédiatement de quel personnage il est ici question, même si, dans la suite de l'article, la référence a disparu et que seul reste le nom propre. On peut avoir affaire à des abréviations, comme dans le cas de l'exemple 3, la "Commission européenne", normalement traduite par Ouzhou Weiyuanhui, est ici abrégée en weihui, mais l'article ne parle que de ce sujet, donc aucun risque de mésinterprétation. Lorsque la personne est inconnue de la majorité des lecteurs, il est possible, dans certains journaux et revues, de mettre d'abord le nom en transcription chinoise, puis, entre parenthèses, le nom en lettres latines, comme dans l'exemple 4. Il peut arriver que seul le prénom soit traduit, comme si l'on pouvait être identifié par lui seul (exemple 6). Certains raccourcis dans les titres d'articles de presse semblent curieux, comme lorsque l'on parle du pape Jean-Paul II en l'appelant Paul II (exemple 8). S'il est parfois difficile pour un lecteur chinois, mal informé des détails de la vie politique, culturelle, économique... étrangère de reconnaître le nom étranger dans sa transcription chinoise, il en est de même pour le lecteur étranger; en effet, il faut souvent faire preuve de vastes connaissances, mais aussi d'une certaine imagination pour reconnaître, par exemple le patronyme irlandais Mac Cartney dans Maikateni. N'oublions pas que les sinogrammes employés ont toujours une signification, c'est l'étrangeté du rapprochement entre des sens normalement exclusifs, qui fait parfois se poser la question : ne serait-ce pas ici un nom étranger? Lequel? 


\section{Conclusion}

La situation morphosyntaxique des noms propres en langue chinoise est donc complexe, selon qu'il s'agit de mots de langue chinoise ou de langues autres, que les règlements administratifs sont ou non suivis par les auteurs de textes, qu'ils soient journalistes, universitaires ou autres. Dans tous les cas, même si le sens n'est pas visible immédiatement, il n'est jamais absent du nom, qu'il s'agisse d'un nom de personne ou d'un toponyme, surtout lorsque c'est un nom propre d'origine chinoise.

\section{BIBLIOGRAPHIE}

Alleton Viviane Les Chinois ou la passion des noms, 1993, Paris, éd. Aubier.

Chen Zhengxiang 陈正祥 中国的地名 (Toponymes de Chine), 1978, Hong-Kong, Shangwu Yinshuguan, $167 \mathrm{p}$.

Chen Zhihua 陈志华 \& Li Yuxiang李玉祥 南溪江中游古村落 (Villages anciens du cours moyen du fleuve Nanxi), 2000, Beijing, Sanlian shudian, 186 p.

Collectif 汉语拼音“中华人民共和国地图”地名索引(Index des toponymes de la Carte de la République populaire de Chine), 1974, Beijing, Ditu chubanshe,153 p.

Collectif 世界地名手册 (Manuel de toponymes mondiaux), 1999, Beijing, Zhongguo ditu chubanshe/Cehui chubanshe, 1335 p.

Collectif 外国地名译名手册 (Manuel de traduction de toponymes étrangers), 1998, Beijing, Shangwu yinshuguan, $910 \mathrm{p}$.

Feng Chengjun 冯承钧 西域地名 (Toponymes des Contrées occidentales), 1980, Beijing, Zhonghua shuju, $159 \mathrm{p}$.

Kadmon Naftali Glossaire de termes pour la normalisation des noms géographiques, 2002, New York, United Nations Publications, 73 p.

Li Yunfu 李运富试论地名标准化“语文标准”的原则问题 (Questions préliminaires sur les principes de « normes écrites » lors de la normalisation des toponymes), in 语文文字应用 Applied Linguistics, 2002-5, pp. 11-19

Liu Jie 刘杰 \& Li Yuxiang李玉祥 泰顺 (Taishun), 2001, Beijing, Sanlian shudian, 154 p.

Xu Jianshun 徐健顺 \& Xin Xian 辛宪 中国姓名文化的奥妙 (Mystères des noms et prénoms chinois), 1999, Beijing, Zhongguo shudian, 472 p.

Revues : 中国地名 (Toponymes de Chine), publiée à Beijing sous l'égide du Ministère des Affaires civiles, et 中国方域。行政区划与地名 (Territoire chinois. Découpage administratif et toponymes), publiée à Taiyuan (Shanxi). 


\section{NOTES}

1. Luxun est le nom de plume choisi par Zhou Shuren.

2. Le terme de « municipalité » s'applique aux quatre plus grandes villes de Chine et au territoire placé sous leur juridiction (Pékin, Shanghai, Tianjin et Chongqing), qui ont le même statut administratif que les provinces ou les régions autonomes ; il désigne aussi des villes et territoires aux niveaux administratifs 2 et 3 .

3. « Glossaire de termes pour la normalisation des noms géographiques », p. 40.

4. Ibid., p. 40.

5. Ibid., p. 42.

6. Ibid., p. 59.

7. «Les Chinois ou la passion des noms », p. 225.

8. Ibid., p. 223.

\section{RÉSUMÉS}

Après une présentation succincte de quelques caractéristiques de la langue chinoise, de son écriture et de la transcription courante, l'auteur nous présente la façon dont sont construits les noms propres de lieux et de personnes, en langue chinoise moderne, qu'il soient chinois ou étrangers .Les toponymes font maintenant l'objet d'une normalisation administrative qui passe en particulier par l'emploi de deux types de mots: les termes génériques et les termes spécifiques, ainsi que par le choix normé de tel caractère pour la transcription de telle ou telle syllabe étrangère.

The author presents some basic characteristics of the Chinese language and Chinese writing, including the pinyin romanization of this language. First of all, there is no space between words, no Capital letters, nothing, to distinguish Proper Names and other kinds of words, except if one uses romanization. Only the place of the word in the sentence, the syntactic function of the word (or phrase), or its meaning, may show that this is a Proper Name. Speaking about Geographical Names, the author divides its presentation into two parts: endonyms and exonyms. The Chinese endonyms follow administrative rules: specific term first, generic term second. The generic terms are mostly made of only one syllable. Rules for writing endonyms in romanization are exposed in details too. The specific and generic terms are separately written, but for names of villages and other small entities. The reality in newspapers and books are different from what is decreted by Governmental organisations: very often the generic term is omitted, this situation may create ambiguity. About exonyms (this category comprises foreign terms as well as terms in minority languages), the situation is complex: one cand find transcription, translation or a mix of both technics. When the foreign Generic term is meaningful, it is usually translated by its Chinese equivalent, the Specific term is translated by Chinese characters whose sound is as near as possible from the original equivalent, there are Administrative norms asking people to use always the same character to write such or such foreign syllable. The Names of persons are usually written with the family name first, the personal name second. The combination of patronyms and idyonyms form most often three syllables, the choice of the personal name is a complicated thing for the parents, because each are meaningful and highly regarded. The foreign names are only transcripted, for a long time there was no rules, each writer following its own 
way, which could create a lot of complications, the Government has these last few years issued regulations for names originally in French, in English, in German, Russian, in Arabic and other languages and writing. What makes reading difficult is the fact that a Chinese character is always meaningful, but one has to forget its meaning to understand the foreign name, either Geographical or Personal.

INDEX

Mots-clés : anthroponyme, chinois, nom de lieu, nom de personne, nom propre, toponyme, transcription

\section{AUTEUR}

\section{PIERRE DE LA ROBERTIE}

Université Rennes 2 Haute-Bretagne 